\title{
Bilateral Cricoarytenoid Joint Fixation in a Patient With Psoriasis
}

Elizabeth V. Joyce (D), medical student, University of Michigan School of Medicine; Beth I. Wallace (D), MD, MSc, Clinical Lecturer, Division of Rheumatology, Department of Internal Medicine, Michigan Medicine; Robert J. Morrison (1), MD, Assistant Professor, Division of Laryngology and General Otolaryngology, Department of Otolaryngology-Head \& Neck Surgery, Michigan Medicine, Ann Arbor, Michigan, USA. Address correspondence to Dr. R.J. Morrison, 1904 Taubman, 1500 E Medical Center Dr, Ann Arbor, MI 48109, USA. Email: morrisor@med.umich.edu. The research study was deemed exempt by the University of Michigan IRB (HUM00185445). Written patient consent was obtained and can be provided on request.

Bilateral cricoarytenoid joint (CAJ) fixation is a rare rheumatologic disorder manifesting with hoarseness, dysphagia, and progressive dyspnea. It is a well-described manifestation of rheumatoid arthritis (RA), ankylosing spondylitis, and vasculitic airway disease such as granulomatosis with polyangiitis, but never with psoriatic arthritis (PsA) $)^{1,2}$.

A 71-year-old man with psoriasis vulgaris and large and small joint pain developed progressive dyspnea, stridor, and hoarseness. Flexible laryngoscopy revealed bilateral vocal cord motion impairment resulting in near complete obstruction of the laryngeal airway, without obvious cause (Figure 1).

Laboratory results were notable for positive antinuclear antibody at a titer of 1:160 with a speckled pattern and elevated erythrocyte sedimentation rate of 21 , but were negative for antineutrophil cytoplasmic antibodies, rheumatoid factor, or anticyclic citrullinated peptide antibodies. He underwent placement of tracheostomy and operative microlaryngoscopy with laryngeal electromyography (EMG) testing, demonstrating normal EMG signal but bilateral CAJ fixation (Figure 2). Given the lack of alternative explanation, the etiology is presumed to be secondary to psoriatic CAJ fixation.

The exact pathogenesis of rheumatologic CAJ fixation is unclear but presumed to be due to CAJ synovitis ${ }^{1}$. Initial treatment may involve steroids, biologic disease-modifying antirheu- matic drugs, and/or voice therapy ${ }^{1}$. Surgical treatment is often used concurrent with medical management or when airway obstruction is present. The most effective surgical treatment is tracheostomy ${ }^{3}$. Alternative surgical treatments are destructive to the vocal cords and carry permanent morbidity in voice quality, with the primary goal of alleviating dyspnea ${ }^{4,5}$. To our knowledge, this is the first reported case of CAJ fixation due to PsA.

\section{REFERENCES}

1. Hamdan AL, Sarieddine D. Laryngeal manifestations of rheumatoid arthritis. Autoimmune Dis 2013;2013:103081.

2. Miller FR, Wanamaker JR, Hicks DM, Tucker HM. Cricoarytenoid arthritis and ankylosing spondylitis. Arch Otolaryngol Head Neck Surg 1994;120:214-6.

3. Bamshad M, Rosa U, Padda G, Luce M. Acute upper airway obstruction in rheumatoid arthritis of the cricoarytenoid joints. South Med J. 1989;82:507-11.

4. Young VN, Rosen CA. Arytenoid and posterior vocal fold surgery for bilateral vocal fold immobility. Curr Opin Otolaryngol head Neck Surg. 2011;19:422-7.

5. Lano CF Jr, Duncavage JA, Reinisch L, Ossoff RH, Courey MS, Netterville JL. Laryngotracheal reconstruction in the adult: a ten year experience. Ann Otol Rhinol Laryngol 1998;107:92-7. 


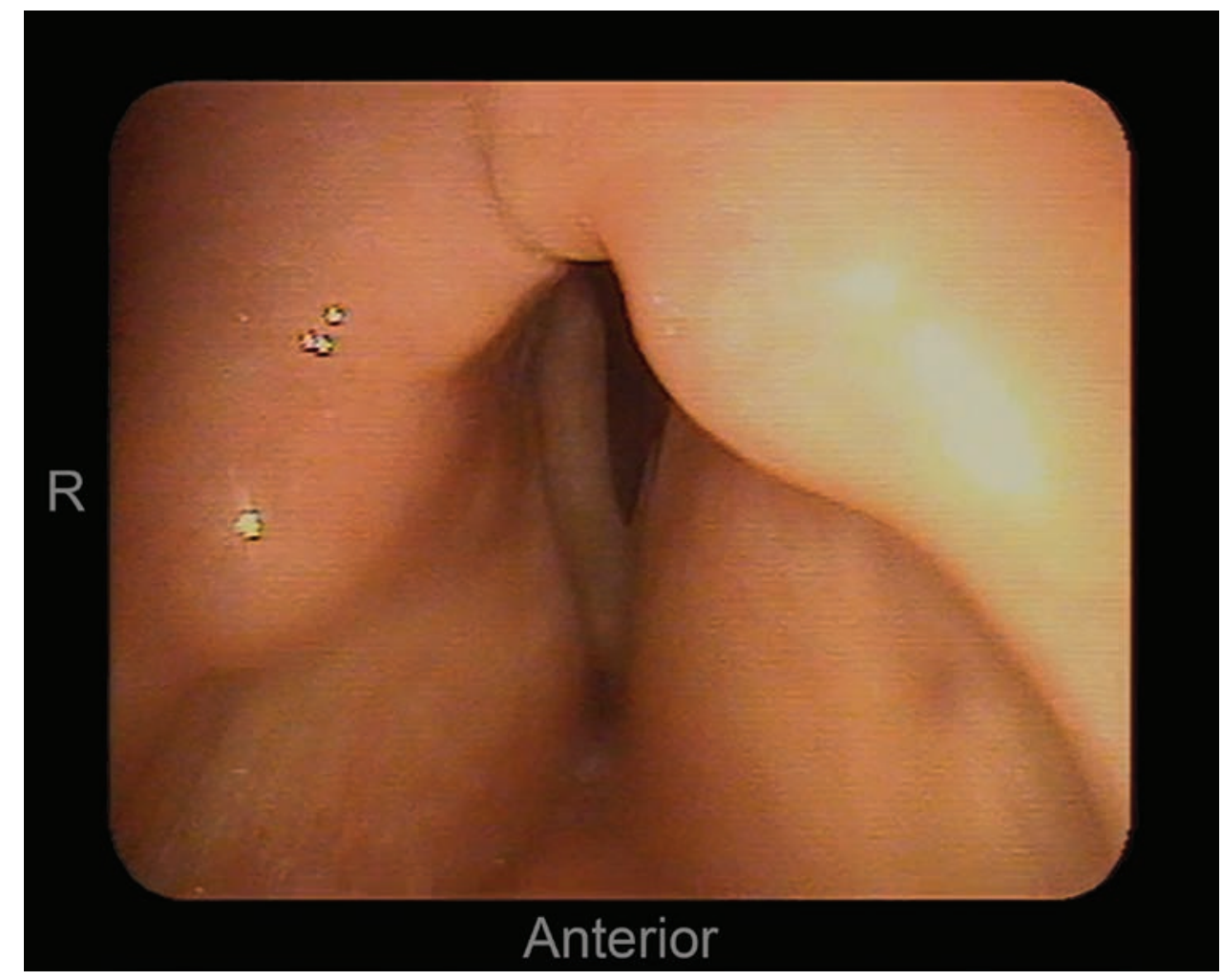

Figure 1. Flexible laryngoscopy performed at referral demonstrating complete right vocal fold immobility and severe restriction in left vocal fold abduction with no evidence of laryngeal trauma or scar.
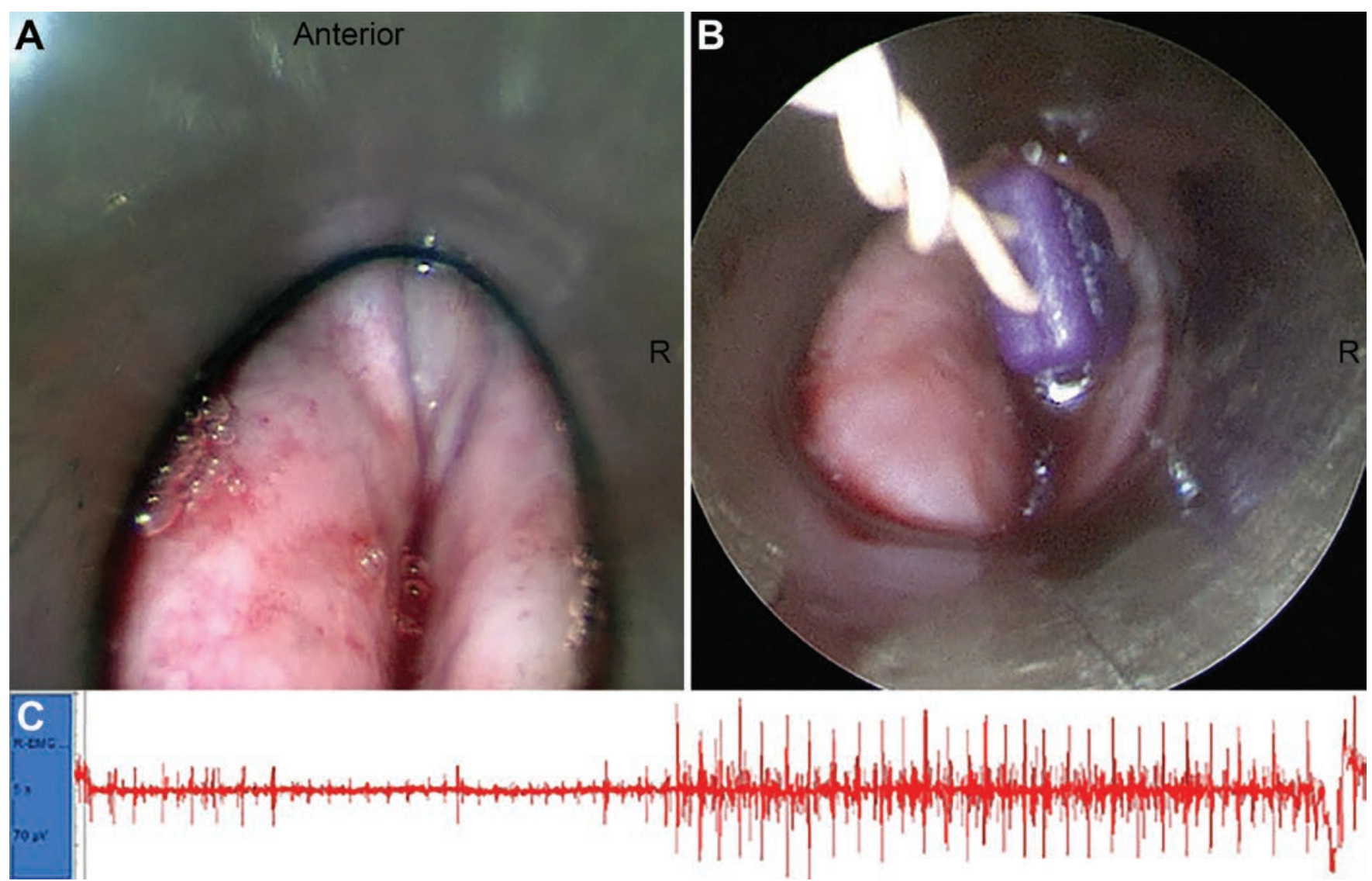

Figure 2. (A) Operative exposure of the posterior glottis demonstrating fixation of the cricoarytenoid joints and absence of intraluminal scar. (B) EMG probe inserted into right vocal fold. (C) EMG results demonstrating normal electrical signal with phasic activity with respiration. EMG: electromyography. 